DOI 10.17816/transsyst201843s1203-211

(C) S. Q. Tang, H. L. Pan, Z. Xu

Maglev Transportation Engineering Technology Research Center

of Tongji University

(Shanghai, China)

\title{
PROGRESS IN THE RESEARCH OF COPPER-OXIDE SUPERCONDUCTORS
}

Since $H \cdot$ Carvalin Onnesse discovered the superconductivity of mercury in 1911 , we have made progress in the research of the superconductor and the superconductor have evolved from single element, alloy to complex compounds with multiple elements. With the development of the research about new superconducting materials, the research of iron based superconductors, copper-oxide superconductor and magnesium boride superconductor is the latest research trend. So far the proved highest superconducting transition temperature of copper-oxide superconductor is $130 \mathrm{~K}$ under normal pressure and could reach more than $160 \mathrm{~K}$ under high pressure. Based on the experience accumulated in past decades, we propose some general introduction about the main structure type, the superconducting principle and the application of copper-oxide superconductor. It is expected that a positive effect would be made in the research of copper-oxide superconductor.

Background: Since H.Carvalin - Onnesse discovered the superconductivity of mercury in 1911, we have made progress in the research of the superconductor and the superconductor have evolved from single element, alloy to complex compounds with multiple elements.

Aim: The purpose of this paper is to explain the differences between copper oxide superconductors and conventional superconductors and their superconducting mechanism.

Methods: The superconducting mechanism and structure of copper oxide superconductors were analyzed by means of literature investigation, conceptual analysis and comparative study.

Results: In this paper, the different structure forms of copper oxide are analyzed, and its superconducting mechanism is described in detail. The applications of several main copper oxide superconductors are introduced.

Conclusion: Based on the experience accumulated in past decades, we propose some general introduction about the main structure type, the superconducting principle and the application of copper-oxide superconductor.It is expected that a positive effect would be made in the research of copper-oxide superconductor.

Keywords: High temperature superconductors, copper-oxide superconductor, superconducting principle, application

\section{INTRODUCTION}

Since 1911, when H. Carmaorin Annes discovered the superconducting properties of mercury, much progress has been made in the research of superconducting materials. In 1986, Bednorz and Mülle, the researchers of IBM company of Switzerland Zurich Institute, found that lanthanum barium copper oxide superconducting state system can be rendered at the temperature 
of $35 \mathrm{~K}$, and high temperature superconductors were discovered. Since the discovery of high temperature copper-oxide superconductor, much progress has been made in the study of superconductivity. Although the mechanism of high temperature superconductivity is still unclear, physicists have put forward various superconducting mechanisms to explain the superconducting mechanism of copperoxide superconductors after years of research.

\section{CRYSTAL STRUCTURE OF COPPER-OXIDE SUPERCONDUCTORS}

Almost all known high temperature superconductors are stacked with perovskite structures.

\subsection{Perovskite structure}

Fig. 1 is a schematic diagram of perovskite structure with two equivalent representations. In fig. (a) the B ion is in the center of the quadrangle of the cube, and the oxygen ion is in the center of the hexagonal surface of the cube; The unit cell in fig.(b) is obtained by moving a distance $a / 2+b / 2+c / 2$ )from the unit cell in fig. (a), (a,b,c are the crystal lattice constant ). The $\mathrm{A}$ ion is in the center, the $\mathrm{B}$ ion is in the four corners of the cube, and the oxygen ion is in the center of the six sides of the cube.

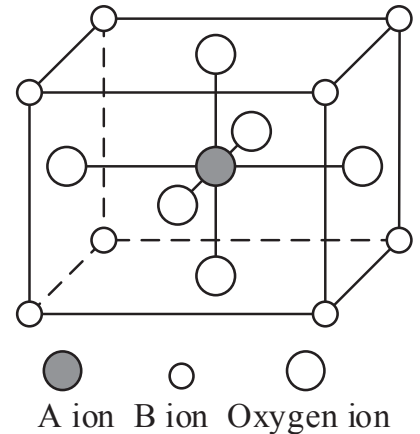

(a)

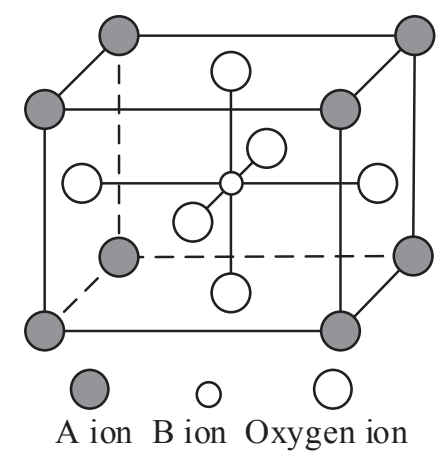

(b)

Fig. 1. Diagram of perovskite structure

\subsection{Perovskite stacking mode}

Fig. 2 shows two stacking patterns of perovskite: One is that one perovskite cell is stacked directly on the other, and the two units share the adjacent A-O surface, which is called rock salt layer. Another way is to put perovskite cells on top of the other. The difference is that the two perovskite cells have a displacement of $a / 2+b / 2$ to reduce electrostatic repulsion and increase the crystal binding energy. The adjacent A-O planes are no longer shared, which is called rock salt block. 

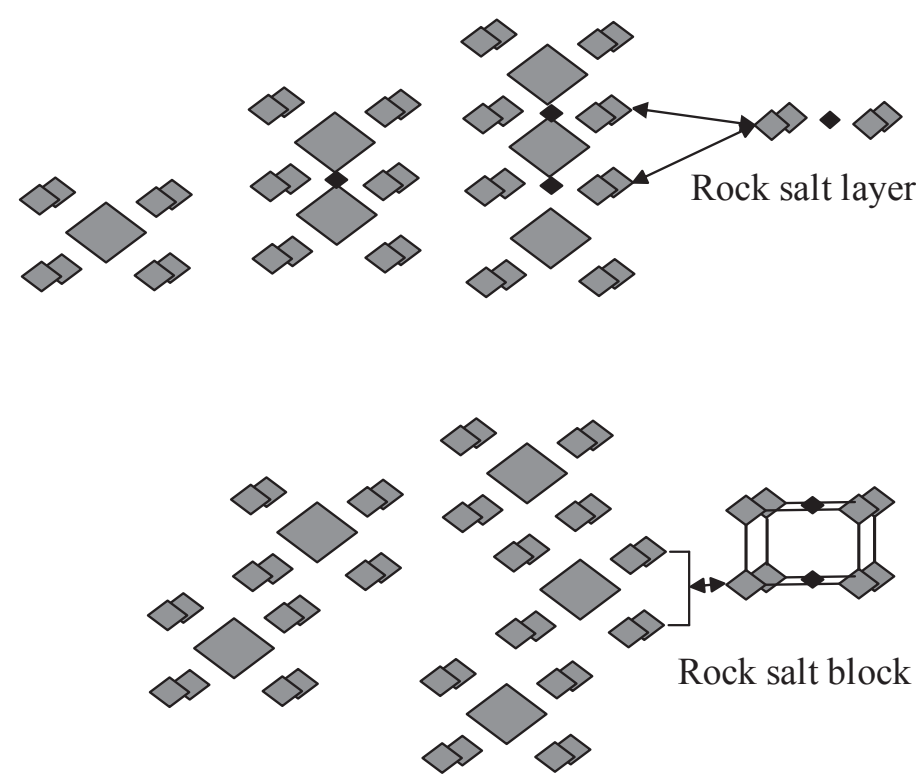

Fig. 2. Perovskite stacking mode

Most high temperature superconductors are stacked in the second mode, which is called R-P structure because it was first proposed by Ruddlesden and Popper. As shown in Fig. 3, the high-temperature superconductor structure is formed by inserting additional layers into the R-P structure, which are often rock salt layers. The general structure can be expressed as $\mathrm{A}_{\mathrm{m}} \mathrm{E}_{2} \mathrm{~B}_{\mathrm{n}-1} \mathrm{Cu}_{\mathrm{n}} \mathrm{O}_{2 \mathrm{n}+\mathrm{m}+2}$ by a fourinteger representation (where $\mathrm{A}, \mathrm{E}, \mathrm{R}$ are various metal ions). In the representation of four integers, the $\mathrm{m}$ is generally the number of layers in the boundary layer at the junction of two units; the 2 is the number of layers adjacent to perovskite, the vast majority of them are two layers; the $\mathrm{n}^{-1}$ is the number of layers intercalated between copper oxygen surfaces and the $\mathrm{n}$ is the number of copper oxygen surfaces.

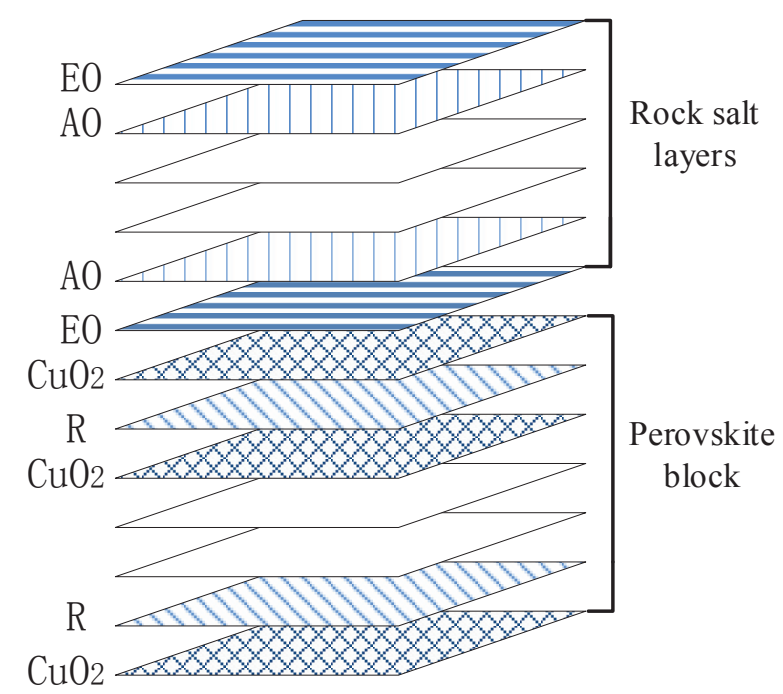

Fig. 3. layered structure of high temperature superconductors and its block method 
After this representation, the physical images of the layered structure are displayed intuitively (Fig. 3). This layered structure can be seen as consisting of two blockss: $(\mathrm{EO})(\mathrm{AO})_{\mathrm{m}}(\mathrm{EO})$ is called the storage reservoir area of electricity storage, also known as rock salt block. $\left(\mathrm{CuO}_{2}\right)\left[(\mathrm{R})\left(\mathrm{CuO}_{2}\right)\right]_{\mathrm{n}-1}$ is called the conductive zone, also known as a perovskite block.

\section{SUPERCONDUCTING MECHANISM OF COPPER-OXIDE SUPERCONDUCTORS}

In explaining the superconducting mechanism of superconductors, we need to understand the causes of resistance and how superconductivity is formed.

\subsection{Resistance causes}

We know that the directional movement of electrons in conductors leads to the generation of currents, and when electrons move between atoms, the vibration of atoms is caused by the electromagnetic force between electrons and nuclei.It is well known that in normal conductors, some electrons are not bound to individual atoms, but can move freely through the lattice of positive ions. When the current is moving through the crystal lattice, especially when the electron in metal collides with the crystal lattice defect, and in the process of motion, it will interact with the crystal lattice vibration and bring about the macroscopic resistance phenomenon. This is the cause of the resistance.

\subsection{Superconducting formation}

By understanding the cause of the resistance, we can know that in order to form superconducting, it is necessary to eliminate the action of electromagnetic force and make the atom eliminate vibration, thus making the resistance zero to form superconducting. The existing scientific research has proved that at extremely low temperatures, the electrons in the atom are running at a low rate of electrons, and this kind of operation is not satisfied with the speed required for its normal operation. This is equivalent to the formation of an electron deficiency, so the core misappropriates adjacent extranuclear electrons, and then all cores continue to misappropriate adjacent electrons, thus forming a phenomenon of external electron sharing. The core takes the public electron as part of the electronic part of itself, and then uses its Coulomb force to transport the extra core electrons. Because of its own role, the electron flow is formed - superconducting current. Thus, the electron flows homewards during the period without interference of other motion forms, so that making its macroscopic resistance zero. 


\subsection{PIntroduction to the classical BCS theory}

The electrons with opposite spins and momentum in metals can be paired to form so-called "Cooper pairs", which can move without loss in the lattice to form superconducting currents. The direct interaction between electrons is a mutually exclusive Coulomb force. If there is only a Coulomb direct action, electrons cannot be paired, but there is also an indirect interaction between electrons mediated by crystal lattice vibration (phonon). The interaction between electrons is attractive to each other, and it is this attraction that leads to the Cooper pair. In general, the mechanism is as follows: when electrons move in the crystal lattice, the positive charges on the adjacent crystal lattice will be attracted, resulting in the local distortion of the crystal lattice points, forming a local high positive charge region. This locally high positive charge region attracts spin opposite electrons, which are paired with the original electrons at a certain binding energy. This binding energy may be higher than the vibrational energy of the crystal lattice atom at very low temperatures, so that the electron pair will not exchange energy with the crystal lattice, and there will be no resistance to form the so-called "superconducting". In traditional superconducting theory, the superconducting critical temperature depends on the interaction intensity of electron pair.

\subsection{Study on Superconducting Mechanism of Copper-oxide Superconductor}

The superconducting mechanism of oxide superconductors is one of the most important topics facing condensed matter physicists. This is because the interaction between electrons in this kind of material is so strong that the normal state electron motion behavior does not seem to be based on the Fermi liquid images of quasiparticle images and energy band theory to understand the knowledge. Although the superconducting state is due to the coacervation of Cooper pairs, many experiments show that the main inducement of the pair is probably not caused by electron-phonon coupling.

As to the mechanism of high temperature superconductivity, it is generally believed that electron pairs appear in the electronic system under the background of magnetic fluctuations, and then superconducting coacervation occurs. The most representative theoretical model is the resonance valence bond model proposed by Anderson, which is called Resonating-Valence-Bond, or RVB model. The RVB model considers that in the spin $1 / 2$ system, the spin state is formed near the reverse spin, while the ground state is the quantum superposition state of the spin singlet, forming the so-called quantum fluctuation liquid (SpinLiquid). These adjacent pairs of spins in opposite directions are in quantum fluctuations, so they are more constrained 
than pure paramagnetic states, and the wave functions describing the opposite pair of spins are similar to the spin parts of the superconducting wave functions of a pair of spin monomorphic pairs. When there is a charge shift in the system, the phase of the spin singlet pair electrons of the RVB ground state is gradually correlated. When the temperature drops below the superconducting transition temperature, phase coherence will be established for the cruise electrons in the system.

It is very difficult to verify the RVB model directly by experiments because the measurement of quantum fluctuations in the RVB ground state leads to a new class of elemental excitations: Spinon (uncharged but with $1 / 2$ spins) and holon (an electron charge but no spin). At present, experimental physicists are trying to find out whether these two new types of meta-excitation exist.

In 2016, physicists at the Brookhaven National Laboratory of the U.S. Department of Energy proposed a theoretical explanation for the high temperature superconductivity of copper oxide complexes. After manufacturing and analyzing thousands of samples of lanthanum strontium copper oxygen (LSCO) mixture, they found that the superconducting critical temperature of the material depends on the concentration of the electron pair in the unit volume of the material.

The magnetic and electrical properties of copper oxide materials were measured by mutual inductance technique to determine the thickness limit of copper oxide materials which can be penetrated by magnetic field. The data can be used to calculate the electron pair density.It is found that there is a good linear relationship between electron pair concentration and superconducting critical temperature. In other words, when there is no free electron pair in the material, the superconducting critical temperature of the copper oxide material should be absolute zero. Specifically, defects in the material prevent the free electron pair from moving, Specifically, defects in the material prevent the free electron pair from moving, leading to the material having to be cooled to a lower temperature to achieve superconductivity. The higher the free electron pair concentration is, the higher the superconducting critical temperature of the material is. According to mainstream superconducting theory, the critical temperature of superconducting depends on the interaction intensity of electron pair, not the concentration of electron pair.Therefore, the correctness of this new theory needs further study.

\section{PROGRESS IN RESEARCH AND APPLICATION OF COPPER-OXIDE SUPERCONDUCTORS}

Copper oxide superconductors have been developed for more than 30 years. The typical high-temperature bismuth superconducting materials $\mathrm{Bi}_{2} \mathrm{Sr}_{2} \mathrm{CaCu}_{2} \mathrm{O}_{8}$ (Bi2212 phase) and $\mathrm{Bi}_{2} \mathrm{Sr}_{2} \mathrm{Ca}_{2} \mathrm{Cu}_{3} \mathrm{O}_{10}$ (Bi 2223 phase) have been widely used 
in the early stage because of their simple molding process and high critical superconductivity temperature.

At present, most of the high temperature superconducting motors (HTS motors) have been fabricated from a Bi 2223 high temperature superconducting material. High temperature superconducting motor has the advantages of high efficiency, large power, small volume and light weight. It is one of the most important applications of high temperature superconducting materials. Currently, several countries, such as the United States, Germany, Japan and China, have developed megawatt motors. Among them, the 36.5 MW HTS synchronous motor for ship propulsion developed by American Superconductor Corporation in 2007 has been delivered to the Navy. The weight of the motor is $75 \mathrm{t}$, which is $1 / 3$ of the mass of the traditional motor of the same power. The torque density is 4 times that of the traditional motor, and it is the highest power HTS motor known at present. The $2 \mathrm{~kW}$ high temperature superconducting claw electrode DC motor developed by Northwest Nonferrous Metals Research Institute of China can be operated in the liquid nitrogen temperature region $(77 \mathrm{~K})$. The motor efficiency is $7 \%$ higher than that of the traditional Z2 DC motor of the same power. However, although bismuth superconducting strips can meet the basic industrial needs, there are still some defects. For example, the $\mathrm{Bi}$ - based superconductor is anisotropic, and the irreversible field in the liquid nitrogen temperature region $(77 \mathrm{~K})$ is only about $0.2 \mathrm{t}$. In addition, the bismuth superconducting strips will use large amounts of precious metal silver. The cost of foundation is too high and is not suitable for mass application. These two limitations limit the application scope of bismuth superconductor.

The most widely used HTS material is YBCO. Compared with bismuth high temperature superconducting material, $\mathrm{YBCO}$ has much higher irreversible field than bismuth superconducting material. YBCO can maintain high critical current density at $77 \mathrm{~K}$ and high magnetic field produced by electric application.

However, the preparation process of YBCO is very complex: firstly, the conductive oxide coating (YBCO layer) is very brittle, the traditional extrusion molding method can not retain its superconducting properties; Secondly, due to the serious problem of weak connection in the YBCO layer, the critical current density of the polycrystal is very low, so it is necessary to eliminate the large angle grain boundary in the current transmission path, which makes it is more difficult in the fabrication process again. The manufacturing process of $\mathrm{YBCO}$ is to fabricate YBCO oxide coating on a flexible metal substrate, so YBCO high temperature superconducting material is also called coating conductor.The coating conductor is mainly composed of three parts: textured baseband, transition layer and superconducting layer, in which the manufacturing cost of textured baseband accounts for more than $60 \%$ of the total cost. 
Shanghai Superconducting Technology Co., Ltd. and Shanghai Jiaotong University Joint Superconducting Research Institute optimize the production process of YBCO tapes. Through the production line autonomy, the cost is greatly reduced, and the finished product rate is greatly increased. At present, it has been used in superconducting motor, HTS magnetic separator, HTS current limiting energy storage and so on. Its structure is shown in Fig. 4. For example: Zhongtian technology superconducting current limiter project is using Shanghai superconducting YBCO strip.

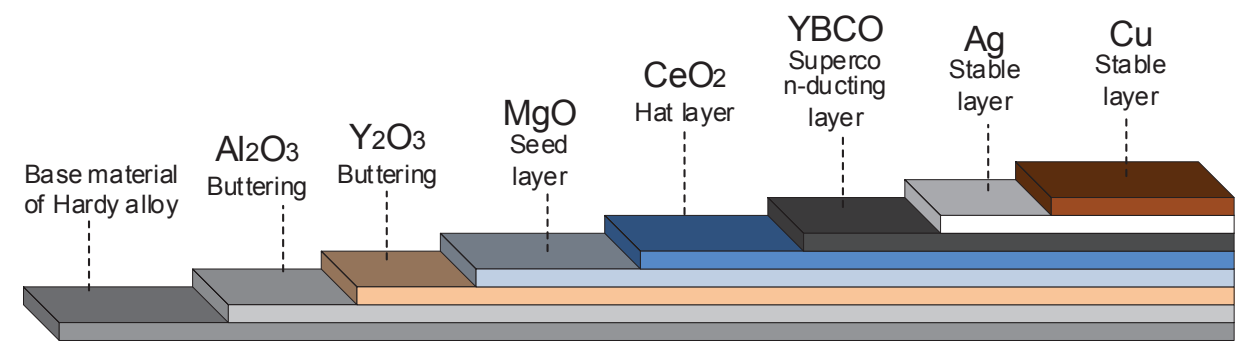

Fig. 4. Schematic show of the deposition process of coating conductor of YBCO

\section{CONCLUSION}

With the development of research, HTS will be widely used in various industries. For example: with the technology and technical problems of production of high temperature superconducting materials gradually resolved, high temperature superconductivity in low voltage high current power transmission transformer, current limiter and superconducting magnetic fluid technology is widely used in application; in addition, the direct use of completely diamagnetic effect and magnetic flux trapping effect of high temperature superconducting materials, new maglev technology will be developed and so on. At present, the development of superconducting materials has been written into "Made in China 2025" and included in the $13^{\text {th }}$ Five-Year Plan of the New Materials Industry. Otherwise, China is promoting the reform of the national science and technology system, changing the national key R \& D projects from the former Ministry of Science and Technology to enterprises. It is believed that HTS will be more and more widely used in the future with the attention and promotion of our country.

\section{ACKNOWLEDGEMENT}

This paper is supported by Hunan provincial science and technology major project, "medium and low speed maglev train integrated technology engineering and high reliability operation demonstration" (Porject No.: 2015GK1001). 


\section{References}

1. Zhang C, Wang F, Li K, Liu K. New Development of practical High temperature Superconducting Materials. Hot Working Technology. 2017;46(14):7-10.

2. Tao H. Preparation of YBCO and exploration of superconducting gap. Zhengzhou University, 2015.

3. Wen HH. Progress in the study of New High temperature Superconducting Materials. Journal of Materials Research. 2015;29(10):241-254.

4. Jia C, Zhang JD, Jin Q. Progress in preparation of High temperature Copper oxide superconductors. Journal of Intraocular Lens. 2014;43(8):2113-2117.

5. Zhang LY. Review of some new developments in high temperature copper oxide superconductors. Low temperature and superconductivity. 2008;11:21-25+33.

6. Wu JB. Finite temperature Landau theory for layered copper oxide superconductors. Journal of Physics. 2006;4:2049-2056.

7. Zhang L, Zhang W. Study on Crystal structure and physical Properties of Copper oxide High temperature superconductors. Journal of Gansu University of Technology. 2000;10(4):104109.

8. Zhang LY, Feng XB. Normal state of high temperature copper oxide superconductors pseudo gap metal state. Chinese Science (series A). 2000;(12):1118-1123.

9. Yuan GS. New Progress in the Application of High temperature Superconducting Materials. Rare metal. 1998;3:2-4.

10. Shen ZH. Electronic structure of copper oxide superconductors. Journal of Physics. 1991;5:278-284.

\section{Information about the authors:}

Shaoqiang Tang, undergraduate degree;

ORCID: 0000-0001-7861-8298;

E-mail:1253630@tongji.edu.cn

Hongliang Pan, associate research fellow, doctor;

ORCID: 0000-0002-2590-6779;

E-mail: panhongliang@tongji.edu.cn

Zhao Xu, associate research fellow, doctor;

ORCID: 0000-0002-4909-6571;

E-mail:1632408@tongji.edu.cn

\section{To cite this article:}

Tang S.Q, Pan H.Q, Xu Z. Progress in the Research of Copper-Oxide Superconductors. Transportation Systems and Technology. 2018;4(3 suppl. 1):203-211. doi: 10.17816/transsyst201843s1203-211 